\title{
Retinopathy of prematurity: age at onset
}

\author{
A R FIELDER, Y K NG, AND M I LEVENE \\ Departments of Ophthalmology and Child Health, Leicester University Medical School, Leicester
}

SUMmARY The age at which retinopathy of prematurity was first seen was determined in 143 infants. In all, the initial ophthalmological examination was normal. Birth weights varied from 630 to $2700 \mathrm{~g}$ and gestational ages from $24 \cdot 5$ to 40.0 weeks. The median postnatal age at which acute retinopathy of prematurity was first seen was 51 and 40 days for those $<28$ and $\geqslant 28$ weeks' gestational age, respectively, and this difference is highly significant. Similar results were obtained when infants were grouped according to birth weight $<1000$ or $\geqslant 1000 \mathrm{~g}$. Using postmenstrual age as the variable, the first signs of retinopathy of prematurity were seen over a fairly narrow age range and $86 \%$ of infants developed retinopathy between 32.5 and 38.5 weeks of age. These findings suggest that the age (but not the occurrence or severity) at which retinopathy of prematurity is first seen is controlled predominantly by stage of development rather than neonatal events.

Despite advances in neonatal care retinopathy of prematurity remains a problem in infants born prematurely. In the United States its incidence seems to be rising, ${ }^{1}$ while in Britian there has been no relevant information since the 1950 s. Surprisingly little attention has been paid to certain aspects of the natural history of this condition and in particular to its time of onset. Silverman and coworkers in 1952 and a report to the Medical Research Council in 1955 both noted that retinopathy of prematurity developed later in babies of lower birth weight..$^{2-3}$ Since that time there have been many changes, not only in the nature of the premature population consequent on improvements in neonatal care but also in methods of ophthalmic examination.

The purpose of this study was to explore the possible relation between the time at which acute retinopathy of prematurity first became visible ophthalmoscopically and postnatal and postmenstrual age. We have analysed retrospectively the records of infants who developed retinopathy of prematurity after an initial normal ophthalmic examination, in whom it was possible therefore to determine with reasonable accuracy the time of onset of the retinopathy.

\section{Patients and methods}

Infants were examined by one observer (ARF) while on one of five neonatal intensive care units in the east midlands of England between January 1981 and April 1985. Infants studied included all infants with birth weights of $1500 \mathrm{~g}$ or less, whether or not supplemental oxygen had been administered, and babies over this weight if they had received additional oxygen. Details of vitamin E supplementation were recorded.

The requirement for inclusion in this study was at least one normal ophthalmic examination before retinopathy of prematurity was observed. For an examination to have been classified as normal adequate visualisation of the retinal periphery was essential; if this was precluded, due to haziness of the media, 'poor view' was recorded. Ophthalmological examination began three weeks postnatally, clinical condition permitting, and was performed weekly until discharge from the neonatal unit. Outpatient follow up was continued as indicated clinically. Pupils were dilated with cyclopentolate $(0.5 \%)$ eye drops instilled 30 and 60 minutes before examination by indirect ophthalmoscopy using a +28 dioptre lens. Since October 1983 an eyelid speculum has been used and the eye rotated by a scleral indentor to enable adequate visualisation of the peripheral retina. Retinal findings were recorded according to the International Classification of Retinopathy of Prematurity; ${ }^{4}$ thus for grade 1 to be diagnosed identification of a demarcation line was essential. It was not possible to record retrospectively the zone of involvement.

\section{Results}

In all, 143 infants satisfied the selection criteria and 
were included in this study. Birth weights ranged from 630 to $2700 \mathrm{~g}$ (median $1090 \mathrm{~g}$ ) and gestational ages between 24.5 and 40.0 weeks (median 29.0 weeks). Fifty three infants in two neonatal units received vitamin $E$ beginning within the first 2 days in nine infants, between 3 and 7 days in 19, between 8 and 28 days in 17 , and after 28 days in two. Vitamin E was given orally in all at a dose of 100 $\mathrm{mg} / \mathrm{kg} /$ day except for six infants, who received 10 $\mathrm{mg} / \mathrm{kg} /$ day. The grade of retinopathy of prematurity at the time of diagnosis was grade 1 in 136, grade 2 in six, and grade 3 in one.

The postnatal age at which retinopathy of prematurity was first noted is represented in the form of a frequency histogram (Fig. 1) and varied between 22 and 85 days (median 45 days), with one exception, that being a $1520 \mathrm{~g}$ infant born at 27.5 weeks' gestation who first showed the retinal changes of retinopathy of prematurity at 118 days. This infant remained well for the first five days of life and received no oxygen. She then developed severe necrotising enterocolitis, with Escherichia coli septicaemia, and required three laparotomies and prolonged intravenous feeding and subsequently died of liver failure at $61 / 2$ months.

The infants were divided into two groups according to their gestational age; $<28$ weeks (50 infants) and $\geqslant 28$ weeks ( 91 infants). There was no record of gestational age for two infants, who were therefore

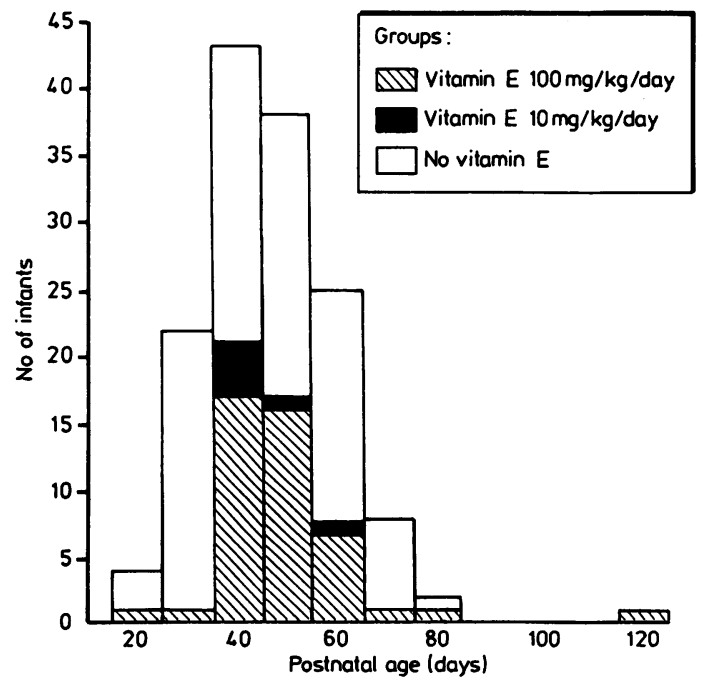

Fig. 1 Frequency distribution of postnatal age at onset of acute retinopathy of prematurity in 143 infants. Hatched and black areas denote those who received supplemental vitamin $E$ (100 and $10 \mathrm{mg} / \mathrm{kg} /$ day, respectively). excluded from this analysis. Figures 2 and 3 represent the postnatal ages at onset of retinopathy of prematurity for each group. Infants with gestational age $<28$ weeks developed retinopathy of prematurity later than those with gestational age $\geqslant 28$ weeks. The 23 infants who were found to have retinopathy of prematurity before 35 days of life were all in the group with gestational age $\geqslant 28$ weeks. The median age at the onset of retinopathy in the group with gestational age $<28$ weeks was 51 days and in the group with gestational age $\geqslant 28$ weeks was 40 days, this difference being highly significant $(p<0.0001$, Mann-Whitney $U$ test). There was a significant negative correlation between gestational age and age at onset of acute retinopathy of prematurity $(\mathrm{p}<0.001)$-that is, the more mature infant developed the retinopathy earlier.

When infants were grouped according to birth weight, $<1000 \mathrm{~g}$ (55 infants) and $\geqslant 1000 \mathrm{~g}$ (88 infants), the results were similar to those obtained for gestational age. The median postnatal age at

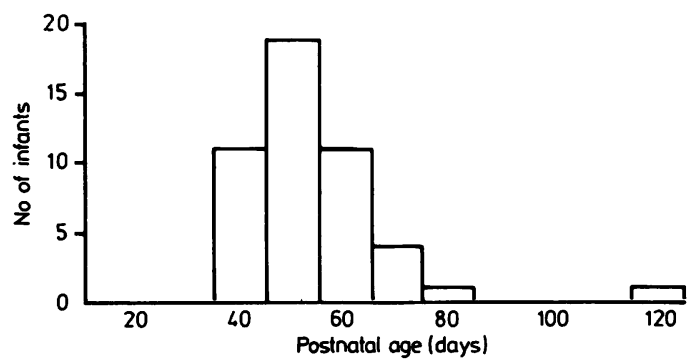

Fig. 2 Postnatal age at onset of acute retinopathy of prematurity in 50 infants $<28$ weeks' gestation.

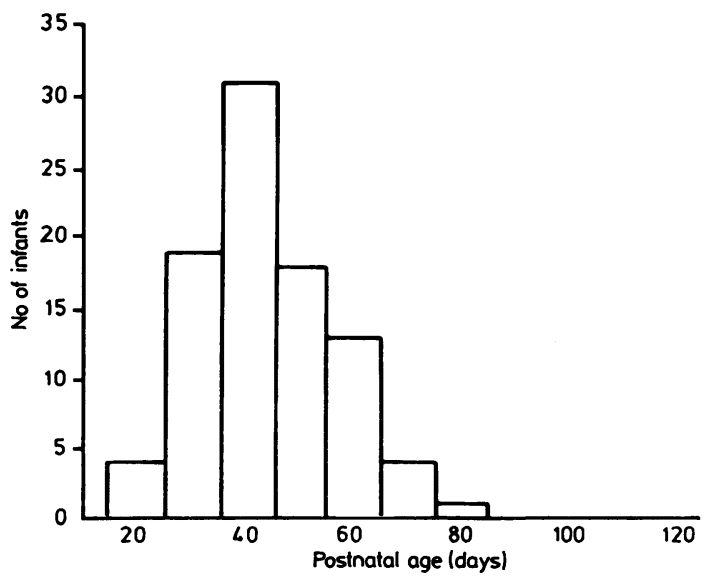

Fig. 3 Postnatal age at onset of acute retinopathy of prematurity in 91 infants $\geqslant 28$ weeks' gestation. 
onset of retinopathy of prematurity for those infants weighing $<1000 \mathrm{~g}$ was 48 days and for those $\geqslant 1000 \mathrm{~g}$ was 40 days. This difference is also significant $(p<0.0001$, Mann-Whitney $U$ test $)$, with infants of heavier birth weight developing retinopathy of prematurity earlier. Taken as a single group there was a significant negative correlation between birth weight and age at onset $(\mathrm{p}<0 \cdot 001)$.

The postmenstrual age at which these infants developed retinopathy of prematurity was determined (Fig. 4). Postmenstrual age was calculated as the sum of the gestational age at birth plus the postnatal age at which retinopathy was first observed. This ranged from 30.8 to 44.5 weeks. Infants with gestational age $<28$ weeks developed retinopathy of prematurity slightly earlier (median 33.7 weeks) than those with gestational age $\geqslant 28$ weeks (median 35.7 weeks). Altogether, 121 infants $(86 \%)$ developed retinopathy of prematurity between 32.5 and 38.5 weeks' postmenstrual age. Four developed the first ophthalmoscopic signs of retinopathy after 40 weeks' postmenstrual age.

The cohort contained 27 infants who were twins. Eleven pairs developed retinopathy of prematurity, but in two pairs one member was not included in the study as retinopathy was present on the first examination. In four of the 11 pairs retinopathy of prematurity affected both twins simultaneously. But for seven pairs (12 infants, retinopathy of prematurity being present on initial examination in two) this was not so, the lighter member being affected first in four. In eight of nine pairs for whom data are available for both members retinopathy of pre-

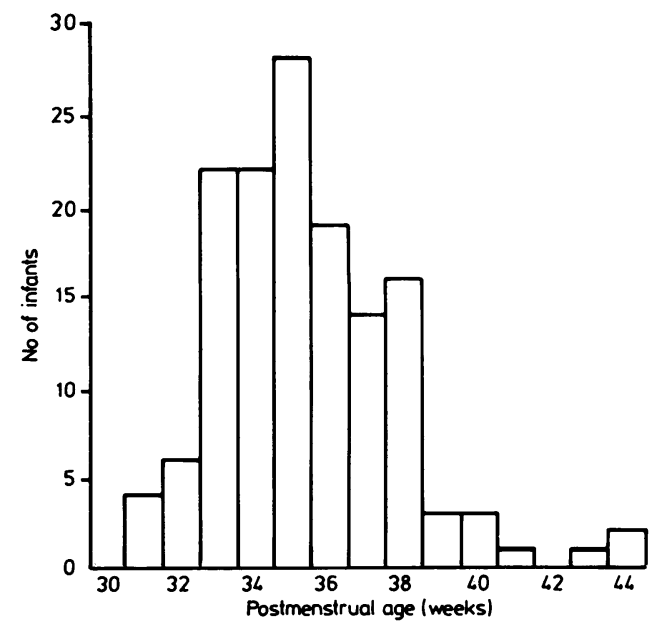

Fig. 4 Postmenstrual age at onset of acute retinopathy of prematurity for all 143 infants. maturity was observed in each member either simultaneously or within two weeks of the other twin. Only one twin was affected in seven pairs, this being the heavier baby in four.

\section{Discussion}

The impetus for this study was the unexpected clinical impression, derived from a few observations, that smaller infants seem to develop acute retinopathy of prematurity later that larger premature infants. We therefore analysed retrospectively the data of 143 infants in whom the initial ophthalmic examination had been normal and consequently in whom the time of onset of acute retinopathy of prematurity could be assessed. Retrospective studies have many defects, but in this instance there was one important advantage in the retrospective nature of the study-namely, there was no observer bias that might influence the eventual outcome.

We have confirmed our impression that the clinical onset of retinopathy of prematurity is later in the highly premature baby compared with his larger counterpart as infants $<28$ weeks' gestation develop retinal changes at a median age of 51 days from birth and those $\geqslant 28$ weeks' gestation at a median age of 40 days, this difference being highly significant $(\mathrm{p}<0.0001)$. The results are similar when infants with birth weights $<1000 \mathrm{~g}$ and $\geqslant 1000 \mathrm{~g}$ are compared, their median ages of onset being 48 and 40 days, respectively. Thus grouping infants according to either their gestational age or birth weight, retinopathy of prematurity develops later postnatally in the more premature infant and in general its onset is inversely related to both of these variables.

By correcting for the degree of prematurity, however, we found that retinal changes develop over a fairly narrow age range, and $86 \%$ developed retinopathy of prematurity in a six week period between 32.5 and 38.5 weeks' postmenstrual age and only $7 \%$ after this time. This may explain the occasional finding of retinopathy of prematurity in the anencephalic ${ }^{5}$ and full term infant. ${ }^{7}$ Furthermore, although retinopathy of prematurity develops later postnatally in small infants, using postmenstrual age as the variable, the position seems to be reversed, those with a gestational age $<28$ weeks developing retinopathy of prematurity at a slightly earlier postmenstrual age than those $\geqslant 28$ weeks. This suggests that the onset of retinopathy of prematurity is hastened in the very small infant, but the two infant groups with gestational ages $<28$ weeks and $\geqslant 28$ weeks are not strictly analogous. The age span is 3.5 weeks in the former and 12.0 in the latter, and both morbidity and mortality are 
greater in the more premature group. Analysis of the infants taken as a single group showed a significant negative correlation between gestational age, birth weight and age at onset.

Inevitably, the assessment of gestational age is approximate, and birth weight exhibiting a scatter for a given gestational age is an even less precise guide to the gestational age of a baby at birth. Ophthalmic examination is difficult, at times potentially hazardous, and for this latter reason performed only once a week. Another important consideration is haziness of the ocular media, preventing visualisation of peripheral retinal details in extremely premature babies, but as relative clarity was achieved before development of retinopathy of prematurity (the criterion for inclusion in this study was an initial normal examination) this aspect could not be responsible for the later postnatal age at diagnosis in the smallest babies. The results we have presented, therefore, should not be interpreted too literally, but we do believe they indicate the time of onset of retinopathy of prematurity as closely as is possible under the circumstances. Furthermore, within the context of this subject, the number of infants in this study is large and sufficient for statistical analysis.

Relatively little attention has been paid to the time of onset of ophthalmoscopically visible retinopathy of prematurity. Silverman and coworkers, studying 76 infants, noted an inverse correlation between the onset of retrolental fibroplasia and birth weight but no significant relation between gestational age at birth and the time of clinical onset of the disease. ${ }^{2}$ In the report to the Medical Research Council by their conference on retrolental fibroplasia it was suggested that 'the more premature the baby at birth the longer delayed, on the average, will be the onset of the retinopathy. ${ }^{3}$ The first Cooperative Study of retrolental fibroplasia reported that 'the age of the infant at onset of retrolental fibroplasia was not dependent on the gestational age or birth weight, but it increased with the length of stay in oxygen. ${ }^{8}$ Commenting on a paper by Palmer, Flynn, using his own data, noted decreasing postnatal age at diagnosis with increasing birth weight. ${ }^{9}$ Recently, Flynn reported that 62 of 74 infants with retinopathy of prematurity were diagnosed between 32 and 40 weeks' postconceptual age. ${ }^{10}$ In his study examinations were performed only fortnightly. Occasionally, retinopathy does not become visible until after 40 weeks' postmenstrual age-as was the case in one infant in the study by Silverman et $a l^{2}{ }^{2}$ 'approximately one-fifth' in the first Cooperative Study, ${ }^{8} 10$ of 74 infants reported by Flynn, ${ }^{10}$ and four of the infants in the present study. Unfortunately, as these articles do not state categorically that all infants were ophthalmoscopically normal before onset of retinopathy of prematurity these data are not ideal to study the age at which retinopathy is first seen. This comment may be over critical, particularly with respect to the study by Silverman in which examinations were performed weekly, ${ }^{2}$ presumably from the first week of life. Studies undertaken before about the mid-1960s cannot be directly compared with more recent reports; the premature population and neonatal management have changed, as have diagnostic criteria and methods of examination, particularly with the introduction of the indirect ophthalmoscope, which permits accurate observation of the peripheral retina.

Vitamin $\mathrm{E}$ was given to 53 of the 143 infants in this study and administered orally in all instances. Advocates of this mode of treatment recommend that it begins intramuscularly within the first two days of life. Vitamin E was started within this period and administered orally in only nine of our infants. Six received $10 \mathrm{mg} / \mathrm{kg} /$ day, a dose not considered to be effective in this context. Quoting two controls and three infants treated with vitamin E, Hittner and coworkers suggested that this substance delayed the development of severe retinopathy of prematurity. ${ }^{11}$ The protective role of vitamin $\mathrm{E}$ in retinopathy of prematurity is still controversial, ${ }^{12}$ and knowing so little about the onset of retinopathy it is clearly inappropiate to speculate as to the possible effect this substance may have.

The pathogenisis of retinopathy of prematurity is poorly understood, but it is generally accepted to be a condition affecting developing retinal vessels. Mesenchymal precursors of the retinal vessels advance centrifugally from the optic disc, reaching the temporal peripheral retina last, just after term. ${ }^{13}$ The degree of prematurity at birth determines how far this process has progressed and consequently the amount of retina vascularised. It is this junction, between the vascularised and non-vascularised retina (the region containing the most recently formed blood vessels), that becomes the site for the development of acute retinopathy. The more premature the infant the more posterior this junction will be and the more posterior the signs of acute retinopathy of prematurity. ${ }^{10}$ As the incidence of retinopathy of prematurity rises with decreasing birth weight ${ }^{14}$ this is the baby particularly prone to develop this condition. Yet this extremely premature infant, often very ill, does not develop retinopathy of prematurity immediately, there being a period of several weeks before the first ophthalmoscopic signs are seen.

We have already alluded to inaccuracies that inevitably exist in these data, but the results 
nevertheless are of interest and raise intriguing questions. Why is there a delay before acute retinopathy of prematurity is first seen? Even more puzzling, why does this response develop later postnatally in the very small, often ill premature infant than in his larger, relatively well counterpart? Our results indicate that the onset of acute retinopathy of prematurity occurs over a fairly short postmenstrual age range, suggesting that a certain developmental stage, probably at a retinal level, has to be reached for the response to evolve. This was suggested by Kinsey. ${ }^{8}$ As already mentioned the retinal vasculature is not fully developed until after term, but it is difficult to postulate that 'immaturity' of these vessels per se can be responsible for the timing of retinopathy of prematurity. If this were so the more immature vessels would be expected to respond early on rather than six to eight weeks postnatally, which is the case. Perhaps it is only after about 32.0 weeks' postmenstrual age that certain enzyme systems or chemical mediators, within the retinal vessel wall or retinal tissues, become active. Further speculation is beyond the scope of this article.

Three aspects in the production of retinopathy of prematurity need to be considered. Firstly, its occurrence, secondly, its severity, both of which are largely determined by gestational age and neonatal events, and thirdly, the age at which retinopathy of prematurity is first seen, which, occurring over a fairly narrow postmenstrual age range, seems to be controlled predominantly by the infant's stage of development and is relatively uninfluenced by neonatal events. Perhaps, therefore, the many factors implicated in the production of this condition act on a system that can only respond when a certain stage of development has been reached and are responsible for the incidence and severity rather than the timing of retinopathy of prematurity. To investigate these factors further a prospective study is under way.
We are pleased to acknowledge the help and encouragement of paediatrician and nursing colleagues in the neonatal units of Leicester, Derby, and Nottingham. Our thanks also to Mr David Shaw for undertaking statistical analyses. Figures were prepared by the Department of Illustration, Leicester Royal Infirmary. Dr $\mathrm{Y} \mathrm{K} \mathrm{Ng}$ is supported by the Medical Research Council.

\section{References}

1 Phelps DL. Retinopathy of prematurity: an estimate of vision loss in the United States-1979. Pediatrics 1981;67:924-5.

2 Silverman WA, Blodi FC, Locke JC, Day RL, Reese AB. Incidence of retrolental fibroplasia in a New York nursery. Arch Ophthalmol 1952;48:698-711.

${ }^{3}$ Report to the Medical Research Council by their council on retrolental fibroplasia. Retrolental fibroplasia in the United Kingdom. Br Med J 1955;ii:78-82.

4 An international classification of retinopathy of prematurity. $\mathrm{Br}$ J Ophthalmol 1984;68:690-7.

5 Addison DJ, Font RL, Manschot WA. Proliferative retinopathy in anencephalic babies. Am J Ophthalmol 1972;74:967-76.

${ }^{6}$ Karlsberg RC, Green WR, Patz A. Congenital retrolental fibroplasia. Arch Ophthalmol 1973;89:122-3.

7 Kushner BJ, Gloeckner E. Retrolental fibroplasia in full-term infants without exposure to supplemental oxygen. Am J Ophthalmol 1984;97:148-53.

${ }^{8}$ Kinsey VE. Retrolental fibroplasia: cooperative study of retrolental fibroplasia and the use of oxygen. Arch Ophthalmol 1956;56:481-529.

9 Flynn JT. Discussion on article by Palmer EA, "Optimal timing of examination for acute retrolental fibroplasia." Ophthalmology 1981;88:667-8.

${ }^{10}$ Flynn JT. An international classification of retinopathy of prematurity: clinical experience. Ophthalmology 1985;92: 987-94.

1 Hittner HM, Speer ME, Rudolph AJ, et al. Retrolental fibroplasia and vitamin $E$ in the preterm infant-comparison of oral versus intramuscular: oral administration. Pediatrics 1984;73:238-49.

12 Schaffer DB, Johnson L, Quinn GE, Weston M, Bowen FW. Vitamin $E$ and retinopathy of prematurity: follow-up at one year. Ophthalmology 1985;92:1005-11.

13 Ashton N. Retinal angiogenesis in the human embryo. Br Med Bull 1970;26:103-6.

14 Flynn JT, Cassady J, Essner D, et al. Fluorescein angiography in retrolental fibroplasia: experience from 1969-1977. Ophthalmology 1979;86:1700-23.

Correspondence to Mr A R Fielder, Department of Ophthalmology, Clinical Sciences Building, Leicester Royal Infirmary, PO Box 65, Leicester LE2 7LX.

Received 8 May 1986 